\title{
High Pressure Freezing of the Aortic Media Retains Relationships of Cells, Collagen and Elastin
}

\author{
D. R. Keene, and S. F. Tufa \\ Micro-Imaging Center, Shriners Hospitals for Children, 3101 S.W. Sam Jackson \\ Park Rd., Portland, Oregon 97239
}

The investigation of connective tissue ultrastructure has historically relied on chemical fixation to stabilize micro architecture. These fixatives are not effective in retaining many matrix molecules, including proteoglycans, and allow precipitation of many other components in subsequent processing steps. Overall tissue shrinkage approaches $30 \%$, and it is clear that there is inequality in shrinkage that occurs with various tissue components. The result is a preponderance of open space between matrix components. Cells shrink considerably and precipitation of molecules in highly concentrated regions leads to artifacts including the lamina lucida and lamina densa of basement membranes.

Cryo-fixation at high pressure followed by freeze substitution seeks to immobilize tissue components within vitreous (non-crystalline) ice, which is later substituted with acetone at temperatures below the re-crystallization temperature of water. Formation of large ice crystals during any part of the preparation protocol significantly distorts structure and can be recognized in the compound microscope. Recognition of smaller freezing artifacts, including microcrystalline ice, is imperative for interpretation of resulting images. However, even when not entirely successful, this preservation technique is far superior to any other methodology of tissue preservation for the electron microscope.

We have been successful in preparing several tissues, including aorta, by high pressure freezing. The overall appearance of these tissues is considerably different from that obtained by conventional methodology, in large part due to the retention of matrix "ground substance". The media of the aorta consists primarily of elastic laminae between which are positioned smooth muscle cells. In chemically fixed aorta, the elastin bundles are seen separated by considerable distance from smooth muscle cells, and appear to be suspended in open space. This is in stark contrast to aorta prepared by high pressure freezing, which demonstrates intimate contact between cells and the elastic laminae. These images offer a "new look" at the aortic media and should be significant in the understanding of cell/cell and cell/matrix interactions. 

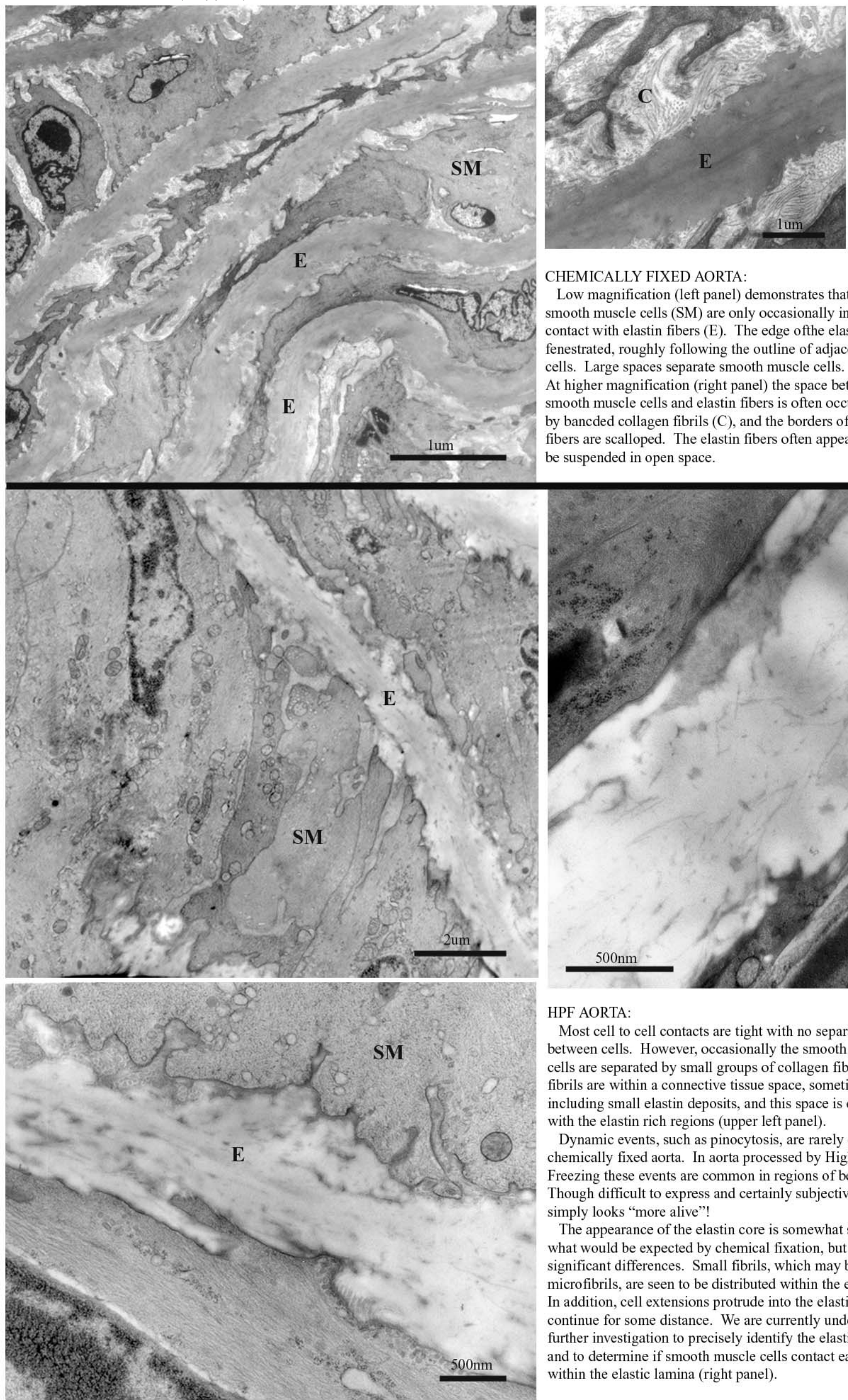

\section{CHEMICALLY FIXED AORTA:}

Low magnification (left panel) demonstrates that the smooth muscle cells (SM) are only occasionally in contact with elastin fibers (E). The edge ofthe elastin is fenestrated, roughly following the outline of adjacent cells. Large spaces separate smooth muscle cells. At higher magnification (right panel) the space between smooth muscle cells and elastin fibers is often occupied by bancded collagen fibrils (C), and the borders of the fibers are scalloped. The elastin fibers often appear to be suspended in open space.

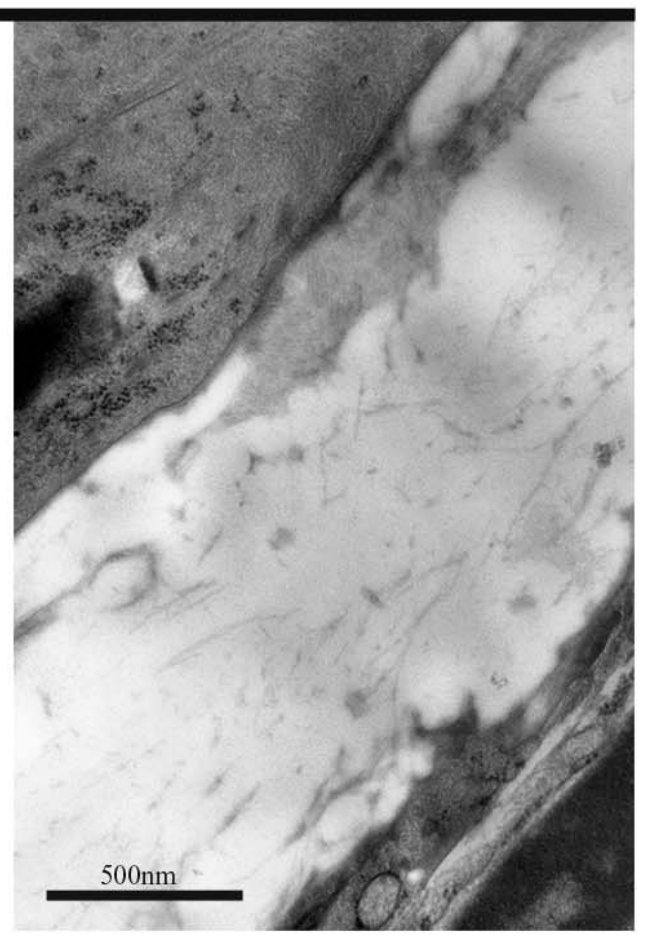

\section{HPF AORTA:}

Most cell to cell contacts are tight with no separation between cells. However, occasionally the smooth muscle cells are separated by small groups of collagen fibrils. These fibrils are within a connective tissue space, sometimes including small elastin deposits, and this space is continuous with the elastin rich regions (upper left panel).

Dynamic events, such as pinocytosis, are rarely captured in chemically fixed aorta. In aorta processed by High Pressure Freezing these events are common in regions of best freezing. Though difficult to express and certainly subjective, the tissue simply looks "more alive"!

The appearance of the elastin core is somewhat similar to what would be expected by chemical fixation, but there are significant differences. Small fibrils, which may be fibrillin-rich microfibrils, are seen to be distributed within the elastin core. In addition, cell extensions protrude into the elastin core and continue for some distance. We are currently undergoing further investigation to precisely identify the elastin inclusions and to determine if smooth muscle cells contact each other within the elastic lamina (right panel). 